\title{
Sub-second dynamic phototuning of alignment in azodendrimer-doped nematic liquid crystal shells
}

\author{
JungHyun Noha ${ }^{\mathrm{a}}$, Venkata Subba Rao Jampani ${ }^{\mathrm{a}}$, Osamu Haba ${ }^{\mathrm{b}}$, Koichiro \\ Yonetake $^{\mathrm{b}}$, Hideo Takezoe ${ }^{\mathrm{c}}$, Jan P. F. Lagerwall ${ }^{\mathrm{a}}$ \\ ${ }^{a}$ University of Luxembourg, Physics and Materials Science Research Unit, 1511 \\ Luxembourg, Grand Duchy of Luxembourg \\ ${ }^{b}$ Department of Organic Materials Science, Yamagata University, 4-3-16 Jonan, \\ Yonezawa, Yamagata 992-8510, Japan \\ ${ }^{c}$ Toyota Physical and Chemical Research Institute, 41-1 Yokomichi, Nagakute, Aichi \\ 480-1192, Japan
}

\begin{abstract}
The alignment of nematic liquid crystal 5CB in micron-thick shells, suspended in and containing aqueous liquid phases, can be rapidly switched between radial (homeotropic) and tangential (planar) director field by doping them with a photoresponsive dendrimer with multiple azobenzene moieties in the branches. The dendrimer spontaneously segregates to the inner as well as outer shell interfaces, folding into an amphiphilic conformation irrespective of the sign of interface curvature. The branches are directed into the liquid crystal, inducing a homeotropic ground state. Upon UV irradiation, the trans-cis isomerization of azobenzene triggers immediate switching to planar alignment. The very fast realignment and the simultaneous response throughout the shell leads to an initially random planar director field, with many topological defects of both positive and negative signs becoming visible within a second of irradiation. All but two +1 defects quickly annihilate, and the remaining defect pair moves up towards the thinnest part of the shell to form the planar steady state. By illuminating with visible light the homeotropic alignment is quickly recovered. By exchanging the solvent used for assisting the dendrimer dissolution, also dynamic phase separation phenomena can be studied in the shells, revealing that the dendrimer solubility in 5CB is greater in the UV-induced cis state than in the trans ground state.
\end{abstract}

Keywords: nematic liquid crystal, azodye dendrimer, photoswitching of alignment, phase separation 


\section{Introduction}

Liquid crystals (LCs) are fascinating liquids, as they combine the molecular mobility and fluidity of regular liquids with the long-range order that is normally only found in solid crystals. This long-range order can be strictly orientational, as in the so-called nematic phase, characterized by rod- or disc-shaped molecules orienting preferentially along a common direction, referred to as the director, abbreviated $\mathbf{n}$. In case of a chiral nematic, also called cholesteric, the chirality leads to a helical modulation of the director along a direction perpendicular to $\mathbf{n}$. In the smectic class of liquid crystals, this orientational order coexists with long-range one-dimensional positional order as well. Numerous fascinating phenomena arise by confining liquid crystals within small spaces, in particular when the boundaries are curved as in spheres or cylinders [1]. The effects of the confinement are dictated by three main components: (1) the shape and scale of confinement, (2) the type and degree of LC order and the related restraints on deformations, and (3) the nature of the interfaces between LC and the surrounding phases, in particular in terms of their effect on LC alignment. To explore variations of the first kind, microfluidic production of droplets or shells $[1,2,3,4,5,6]$ or spinning of fibers with liquid crystals $[1,7,8,9]$ are highly useful, and the second aspect can be explored relatively easily by selecting different liquid crystal-forming substances and heating or cooling near or past phase transitions, for instance between nematic and smectic phases $[10,11,12,13]$. The third aspect is more challenging to vary at will since the interface is often formed with the same phase that creates the confinement, or with stabilizers that are needed to avoid merging of many small LC volumes into fewer larger ones [14].

A very interesting tool for dynamically tuning the alignment is lightsensitive surface-active agents (surfactants) containing azobenzene moieties [15]. Upon illumination by UV light, azobenzene changes its shape from the rod-shaped trans isomer to the kink-shaped cis isomer, and the reverse transformation can be induced by exposure to visible light. A surfactant typically contains one polar end and one non-polar end, hence, when introduced into an LC confined by a more polar phase the surfactant segregates to the interface, with the non-polar section pointing into the LC and the polar end directed outwards, into the confining phase. If the azobenzene unit is in- 
troduced appropriately, the photoisomerization may change the interaction of the non-polar section with the LC, in this way enabling photoswitching of the LC alignment. A good example is the dendrimer LCD2-8azo6 $[16,17,18,19,20]$, in the following abbreviated AzD (Azo Dendrimer), see Fig. 1a. In an LC host phase in contact with a polar environment (liquid or solid), AzD segregates to the interface where it folds in an amphiphilic conformation, the non-polar side pointing into the LC. Here it induces homeotropic alignment (director perpendicular to the interface) as long as it is not exposed to UV light. In contrast, upon UV irradiation the kinking of the azobenzene moieties changes the interaction with the liquid crystal such that planar alignment (director in the interface plane) is promoted, see the schematic in Fig. 1b. The reverse switching, back to homeotropic, can be triggered by irradiation with visible light. A very fast azobenzene response on the order of picoseconds can induce a rapid alignment change in the millisecond range, even in fairly viscous liquid crystals with long-range orientational order.

So far this photoswitchable alignment agent was used on nematic liquid crystals confined by flat interfaces [16, 17, 18], in nematic droplets [18], and with the LC surrounding solid glass spheres [19] or rods [20]. The LC in these studies makes boundaries with zero, positive and negative curvature, respectively. A configuration that combines interfaces with positive as well as negative curvature is that of a liquid crystal shell (Fig. 1c-d), in which a thin spherical layer of LC finds itself surrounded by aqueous phases both on the inside and on the outside. Until now, no LC shells were prepared with $\mathrm{AzD}$, hence an interesting question is whether the dendrimer can be used to dynamically switch the alignment of LCs also in shell topology. In this paper, we explore this potential by preparing LC shells of the common nematogen $5 \mathrm{CB}$ doped with $\mathrm{AzD}$ and studying their response to illumination by UV and visible light. We confirm that AzD can indeed be used to modulate the alignment in the shell very rapidly. Applying the concept to LCs with more complex phase sequences, including LCs forming cholesteric or smectic phases, this will open prolific new avenues for studying pattern formation, generation and annihilation of topological defects, and the response of the LC to different types of imposed frustration [1]. A further interesting observation is that even small changes to the solvent used for assisting AzD dissolution in 5CB can lead to partial phase separation when AzD is in its ground state, while the dendrimer in its photo-induced cis state appears uniformly dissolved in the host. 
(a)
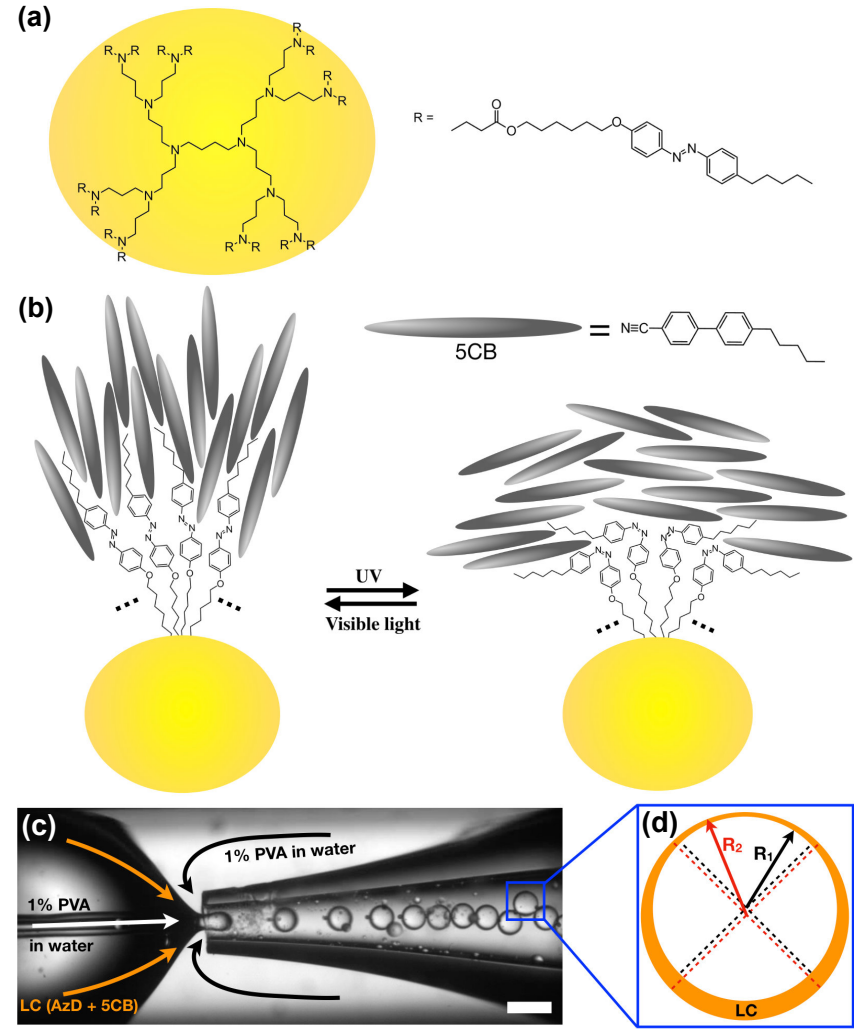

Figure 1: (a) Chemical structure of the azodendrimer LCD2-8azo6, here abbreviated AzD. (b) Expected AzD conformation change upon UV illumination, and its effect on the alignment of nematic 5CB. For simplicity, the 5CB mesogens are depicted as ellipsoids and only 4 out of the 16 azobenzene moieties of $\mathrm{AzD}$ are depicted, ignoring the 3D extension of the molecule. (c) Microfluidic production of AzD-doped 5CB shells (heated to isotropic phase). (d) Schematic drawing (cross-sectional view from the side) of the asymmetric nematic shell with inner radius $R_{1}$ and outer radius $R_{2}$. The average thickness $(h)$ is measured by subtracting $R_{1}$ from $R_{2}$. Scale bar is $200 \mu \mathrm{m}$. 


\section{Experimental details}

We prepare mixtures by adding AzD to 5CB (4-cyano-4'-pentylbiphenyl, purchased from Yantai Xianhua Chem-Tech Co. Ltd., China) at 0.1, 0.3, 0.6 and 1 wt.- \%, respectively. The dissolution is assisted using either chloroform (Sigma-Aldrich) or dichloromethane (DCM, Sigma-Aldrich), added at about 1:1 volume ratio. The dissolution is further supported mechanically by a stir bar rotating in the sample. After the mixture appears uniform to the eye (after about one hour of stirring), the assistive solvent is evaporated at $80^{\circ} \mathrm{C}$ (chloroform) or at $40^{\circ} \mathrm{C}(\mathrm{DCM})$, for two days with continuous stirring. If the solvent is not sufficiently removed, it can be traced by shells collapsing immediately after production: due to continuous diffusion of residual solvent away from the shells, their interfaces become unstable, resulting in shell rupture. In some cases an annealing step, up to several months long, was added between the evaporation of the assistive solvent and the production of the shells. The investigation of the shells was always done immediately after their production.

A coaxial capillary microfluidics device is prepared by cylindrical and square glass capillaries (purchased from Vitrocom and Harvard apparatus, respectively). Two tapered cylindrical capillaries are inserted into a square capillary and the sharp tips are facing each other in the middle of the square capillary, see Fig. 1c. We use 1 wt.-\% polyvinyl alcohol (PVA, Mw 13,00023,000 $\mathrm{g} \mathrm{mol}^{-1}, 87-88 \%$ hydrolysed, Sigma Aldrich) in water solution as the inner and outer phases in a suspension of shells. The inner fluid is flown through a narrowly tapered cylindrical capillary (an injection capillary), as shown in Fig. 1c. The AzD and 5CB mixture, flown as the middle fluid, is introduced through the void between the cylindrical and square capillaries, and the outer fluid is injected into the same void from the opposite direction. The coaxial inner+middle fluid flow becomes flow-focused by the outer phase into the tip of the collection capillary, resulting in a stream of shells when the inner+middle flow breaks up due to the Rayleigh-Plateau instability.

For polarizing optical microscopy investigations (Olympus BX-51) we fill a rectangular capillary with a shell suspension. A UV source at $365 \mathrm{~nm}$ (UVATA LED UV system, $8800 \mathrm{~mW} \mathrm{~cm}^{-2}$ at full power at the exit of the light guide) is used for the trans-cis photoswitching. The illumination is about 5 $\mathrm{cm}$ above the sample and at $45^{\circ}$ to the sample plane, hence full LED power corresponds to about $350 \mathrm{~mW} \mathrm{~cm}^{-2}$ at the sample plane. For promoting the cis-trans back isomerization the sample is exposed by white light, provided by 
temporarily increasing the microscope illumination to $100 \%$ power. For normal imaging, we illuminate the sample with $50 \%$ intensity of the microscope lamp, which does not affect the AzD on the timescale of the experiments, about 5 minutes. In contrast, 100\% lamp power leads to immediate cis-trans back isomerization. Since the standard room lighting would also trigger the back relaxation to the trans isomer state, we have carried out all experiments in a room with yellow light. The nematic director configuration is observed using the polarization optical microscope as well as with fluorescence confocal polarization microscopy (FCPM, Nikon A1R+).

For the latter investigations the $5 \mathrm{CB}+\mathrm{AzD}$ mixture is stained with 0.01 wt.- $\%$ of a dichroic fluorescent dye, 7-diethylamino-3,4-benzophenoxazine-2one (Nile red, Sigma-Aldrich). The dye molecules tend to orient along the LC director due to their shape anisotropy [21, 22], which allows analysis of the director field and topological defects therein under polarized laser excitation. The cells for FCPM imaging are made using a $160 \mu \mathrm{m}$ thick cover glass on one side and a $1.1 \mathrm{~mm}$ thick microscope slide on the other. The thin side is coated with teflon in order to prevent sticking to the glass of the AzD-doped shells. The cell thickness is set to $300 \mu \mathrm{m}$ using a transparent plastic film (3M, CG3300). A laser with wavelength $561 \mathrm{~nm}$ excites the Nile red, and the emission is detected in the spectral region of 600-670 nm. Any fluorescence from $\mathrm{AzD}$ is negligible under these conditions. The excitation laser polarization is along the horizontal plane in Fig. 6 and 7. While carrying out the confocal imaging of samples in the UV-induced cis state, we applied a weak continuous UV illumination $\left(25 \mathrm{~mW} \mathrm{~cm}^{-2}\right)$ throughout the imaging process for avoiding AzD back relaxation to trans isomer state induced by the excitation laser.

\section{Results and discussion}

\subsection{Photoswitching of $5 C B$ shell alignment}

Shells made from homogeneous mixtures with 1\% AzD in 5CB show a regular homeotropic texture in the ground state (Fig. 2a and Fig. 3a), indicating that the dendrimer segregates to both the in- and outside, thereby controlling the alignment throughout the shell. The smooth and uniform ground state texture confirms adequate dissolution of AzD in the 5CB host. This can be ensured soon after evaporating the assistive solvent when chloroform is used (the shells in movie SI1 and Fig. 3 were prepared this way), whereas mixture preparation assisted by DCM yields phase separated shells 


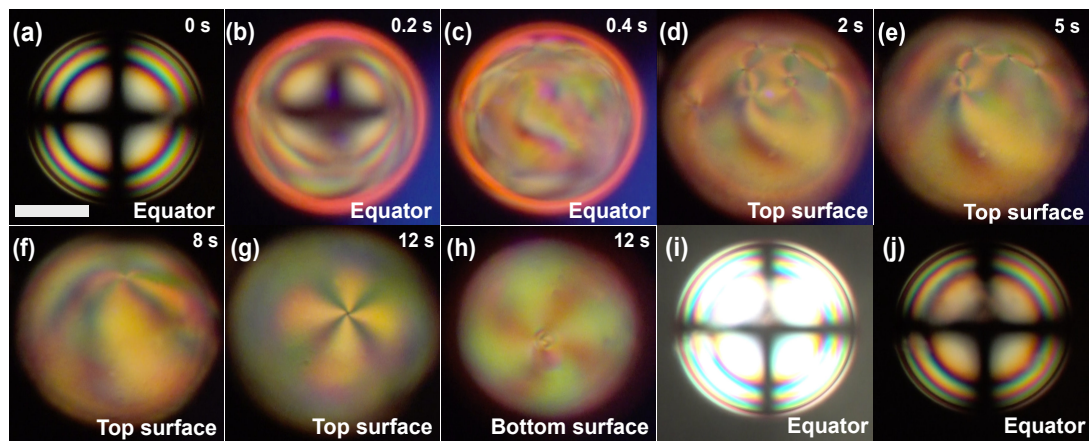

Figure 2: POM images of a nematic shell with well dissolved 1 wt.- $\%$ AzD, prepared using DCM as assistive solvent, followed by 1 year of annealing before shell preparation. Panel (a) is before UV illumination (homeotropic alignment), and (b-h) are during UV illumination as a function of time. Under white light illumination achieved by increasing the microscope illumination (i), the shell turns back to homeotropic after exposure for 10 seconds (j). As indicated in the figure, the focus is changed between the top, equator and bottom of the shell. The shell diameter $2 R_{2}=120 \mu \mathrm{m}$ and the average thickness $h=3 \mu \mathrm{m}$. Scale bar is $50 \mu \mathrm{m}$.

if they are produced soon after mixture preparation (to be discussed in Section 3.2). Homogeneous dissolution using DCM is possible, but it requires very long annealing time between solvent evaporation and shell production, on the order of months; the shell in Fig. 2 and movie SI2 was made from a DCM-assisted mixture one year after preparation.

Upon UV illumination the texture changes (movies SI1 and SI2 show the complete process) to non-uniform planar within a fraction of a second (Fig. 2b-c). As can be expected upon such rapid alignment switching, the di-
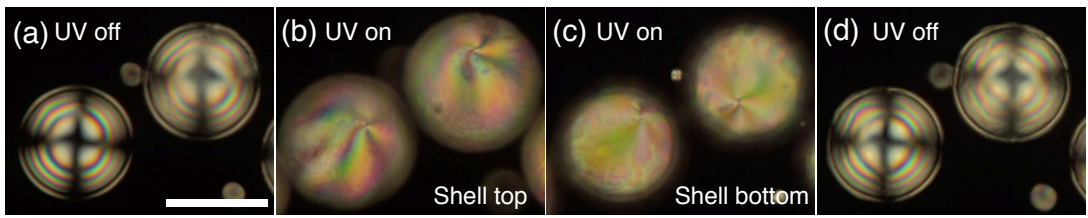

Figure 3: POM images of a nematic shell with well dissolved 1 wt.- $\%$ AzD, prepared using chloroform as assistive solvent, the shells being prepared soon after solvent evaporation. Panel (a) is before UV illumination (homeotropic alignment) whereas (b-c) are during UV illumination. Under white light illumination achieved by increasing the microscope illumination to twice the regular observation intensity, the shell turns back to homeotropic after exposure for about 10 seconds (d). The shell diameter $2 R_{2}=105 \mu \mathrm{m}$ and the average thickness $h=5 \mu \mathrm{m}$. Scale bar is $100 \mu \mathrm{m}$. 
rector field is initially highly non-uniform, with a string of topological defects distributed around the shell (Fig. 2d-e). Since, according to the PoincaréHopf theorem [1,3], the topological defect sum across a planar shell interface is required to be +2 , we can conclude that negative as well as positive-signed defects are formed. Indeed, over the course of the first 15 seconds after turning on UV illumination all but two +1 defects annihilate (Fig. 2d-f). These are initially roughly antipodal, one at the shell top (Fig. $2 \mathrm{~g}$ and $3 \mathrm{~b}$ ) and one at the bottom (Fig. $2 \mathrm{~h}$ and $3 \mathrm{c}$ ), but as described below, further annealing moves both defects up close to the thinnest point of the shell. The motion of defects is slower than in pure $5 \mathrm{CB}$ shells, suggesting that the presence of 1 wt.- $\%$ AzD leads to a non-negligible increase in viscosity.

We subsequently expose the sample to white light by increasing the intensity of the microscope illumination. As a consequence, the ground state homeotropic alignment is brought back via the cis-trans back isomerization of the azobenzene moieties (Fig. 2i-j and 3d). The AzD-driven alignment change in shells is thus reversible and the sequential change can be achieved in the order of seconds.

An interesting observation is that the topological defects seen in the UVinduced planar state are always of integer strength, whereas shells that have a planar ground state often exhibit half-integer defects (see below and references such as $[1,3,4,5])$. At present, we can only speculate about the reason for the absence of half-integer defects. The movies show some irregularities in the texture during switching, suggesting that some small aggregates of AzD may still exist, or that the change from homeotropic to planar orientation is not quite simultaneous throughout the shell. Local aggregates as well as local variations in alignment could both seed defects of integer strength and this may thus be the reason why half-integer defects are not observed. Another possibility is that the in- and outsides of the shell do not switch exactly simultaneously, which would thus yield a brief hybrid-aligned (planar on one side, homeotropic on the other) intermediate state. The bend in the director field that mediates hybrid alignment prohibits the formation of half-integer defects [5], hence this could be another explanation why only integer defects are seeded.

The fast alignment switching made possible by AzD makes it a highly useful alignment agent for LC shells. The photoswitching is independent of temperature and it is very fast, allowing us to change from homeotropic to planar alignment in the shell, and back, in seconds. This enables the resulting defect formation and annihilation described above, in contrast to 
previous studies of alignment change in LC shells, which were much slower. Lopez-Leon and Fernandez-Nieves exchanged the continuous phase from a PVA solution to a surfactant solution, changing the outer alignment from planar to homeotropic [23], and Noh et al. studied shells prepared with surfactant on one side and PVA on the other over long enough time for the surfactant to diffuse through the shell [14]. The former study took the shell from uniformly planar to hybrid, whereas the latter took it from hybrid to uniformly homeotropic. In neither case could both interfaces be switched and both procedures take long time. In contrast, AzD allows us to switch both shell interfaces within a fraction of a second.

\subsection{Phase separation in 5CB shells with incompletely dissolved azodendrimer}

While most prior studies with AzD-doped LCs used mixtures prepared with chloroform as assistive solvent, we here also studied the effect of using the very similar solvent dichloromethane (DCM). Although the mixtures appear homogeneous to the eye, preparation of shells after evaporating the DCM reveals that they are, in fact, heterogeneous, and they remain so over very long times. Shells made soon after mixture preparation show clear signs of phase separation, even at AzD concentrations as low as 0.1 wt.- $\%$, as shown in Fig. 4, 5 and 7. It is remarkable that this rather minor change in solvent can have such a dramatic effect, in particular as both solvents were evaporated before the shells were prepared. It appears that the exchange of one chlorine atom for a hydrogen makes DCM so much worse as a solvent for $\mathrm{AzD}$, compared to chloroform, that the dendrimer is partially collapsed into a globule state in DCM solution. This would make it less accessible for $5 \mathrm{CB}$ contact compared to the more swollen state in chloroform, allowing the dendrimer to stay partially phase separated over very long times.

For 0.1-0.3 wt.- $\%$ AzD concentration the ground state alignment is dominated by the aqueous PVA solution, thus remaining planar before, during and after illumination, as witnessed by the presence of topological defects with a total charge of +2 . In the shell in the left column of Fig. 4 there are two +1 defects near the thinnest point of the shell, whereas the shell in the right column has one +1 defect and two $+1 / 2$ defects. Further variations are shown in the Supporting Information. Interestingly, the small increase from 0.1 wt.- $\%$ to 0.3 wt.- $\%$ AzD concentration between the left and right columns in Fig. 4 gives rise to a striking textural color difference. The shells in the two columns differ only slightly in diameter and they have about the same thickness, hence the color should be similar, yet the shell with less AzD has 


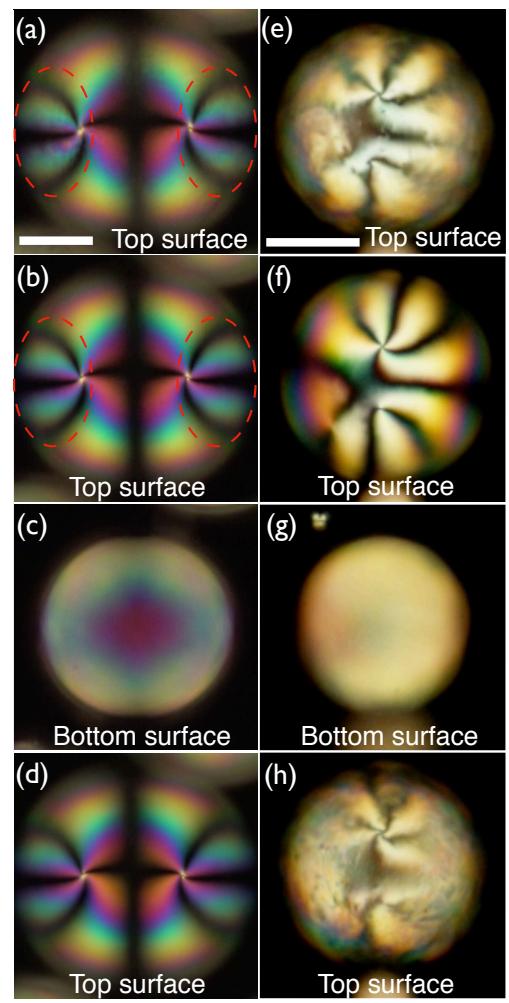

Figure 4: Left column: 0.1 wt.-\% AzD; right column: 0.3 wt.-\% AzD. POM images of nematic shells produced with freshly prepared mixture with DCM as assistive solvent, before UV illumination with focus at the shell top (a/e), during UV illumination with focus on shell top (b/f) and on shell bottom (c/g), after UV illumination with focus on shell top $(\mathrm{d} / \mathrm{h})$. The red dashed circles in (a) and (b) highlight the disappearance of perturbed texture upon UV irradiation. The shell diameter $\left(2 R_{2}\right)$ is $140 \mu \mathrm{m}$ in (a-d) and $110 \mu \mathrm{m}$ in (e-h). The average thickness $h=4 \mu \mathrm{m}$ in both columns. Scale bars are $50 \mu \mathrm{m}$.

strong purple to green colors belonging to the first order of the Michel-Lévy diagram, whereas the shell with 0.3 wt.- $\%$ AzD is dominated by a pale yellow color, reflecting a considerably smaller phase retardation, further to the left in the Michel-Lévy diagram. We interpret this as a sign of the disordering effect of the inadequately dissolved $\mathrm{AzD}$, reducing the nematic order parameter of $5 \mathrm{CB}$ and thus its birefringence, in addition to causing the perturbed texture.

Interestingly, a photoswitching effect could still be observed even in these shells with inadequately dissolved AzD, namely with respect to the phase separation: it appears that the photoisomerization of the azobenzene moieties 


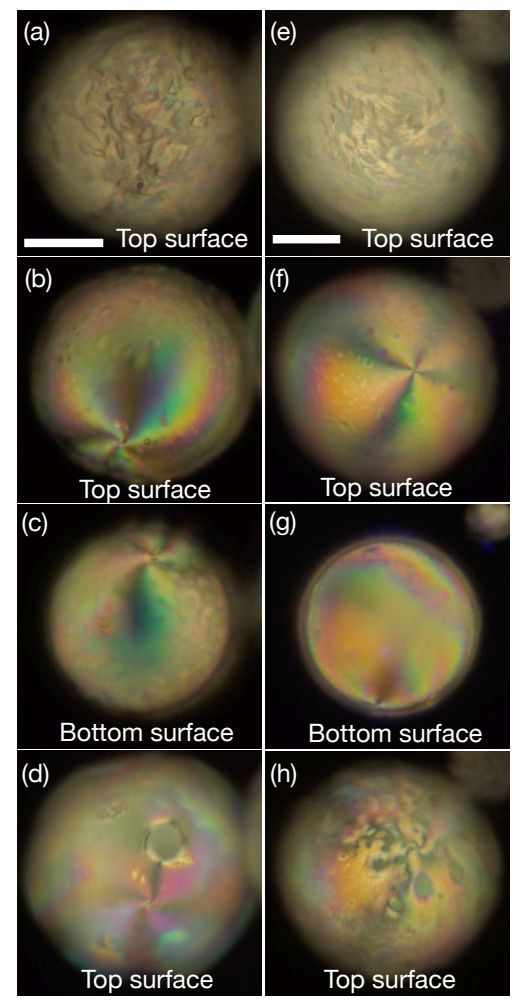

Figure 5: Left column: 0.6 wt.- $\%$ AzD; right column: 1 wt.- $\%$ AzD. POM images of nematic shells produced with freshly prepared mixture, using DCM as assistive solvent, before UV illumination with focus at the shell top (a/e), during early stages of UV illumination with focus on shell top (b/f) and on shell bottom (c/g), after short (30 s) white light illumination with focus on shell top $(\mathrm{d} / \mathrm{h})$. The shell diameter $\left(2 R_{2}\right)$ is $130 \mu \mathrm{m}$ in both columns, and the average thickness $h=5 \mu \mathrm{m}$ in (a-d) and $h=3 \mu \mathrm{m}$ in (e-h). Scale bars are $50 \mu \mathrm{m}$.

assists the AzD dissolution in 5CB. At 0.1 as well as at 0.3 wt.- $\%$ AzD, the pristine shell shows a perturbed texture indicative of phase separation (Fig. 4a and e), but during UV irradiation the texture gets much smoother, suggesting that the dendrimer distributes more uniformly around the shell in the cis isomer state. After the UV irradiation is terminated, the smooth texture stays in case of 0.1 wt.- $\%$ AzD (Fig. 4d) whereas the perturbed texture returns for 0.3 wt.- $\%$ dendrimer (Fig. 4h).

At an AzD concentration of 0.6 wt.- $\%$ or greater, we do find photoinduced alignment switching even in case of non-homogeneous mixtures. However, the ground state is not homeotropic but shows an ill-defined texture with many 
signs of phase separation, see Fig. 5. Upon UV illumination (movie SI3) the texture again becomes much more uniform, with clear planar alignment, confirming the results from Fig. 4 that the tendency to phase separation is reduced in the cis state of the azobenzene moieties. While the UV light is on, two +1 defects can be clearly resolved, one on the top and one on the bottom shell side, for 0.6 wt.- $\%$ as well as for 1 wt.- $\%$ AzD, see Fig. 5b-c and f-g. After turning off the UV illumination (Fig. 5d/h) the texture becomes more inhomogeneous again, but the phase separation is not as strong as prior to photoisomerization. Homeotropic alignment is never seen when the AzD is insufficiently dissolved.

We also investigated shells of this type using FCPM, see Fig. 6-7. As seen in the polarizing microscopy mode image in Fig. 6a, the initial texture of the shell, here with 0.6 wt.- $\%$ AzD incompletely dissolved, is highly irregular with clear signs of phase separation. This impression is corroborated by the FCPM image prior to UV illumination (Fig. 6b), which even suggests a certain unevenness in the shell surface. This might be expected in case of islands of partially collapsed AzD globules separated by more or less pure $5 \mathrm{CB}$.

As the UV irradiation is started, the FCPM imaging immediately confirms the almost complete disappearance of islands of phase separated AzD upon photoisomerization of the azodendrimer, see Fig. 6c. While some areas of uneven surface can be recognized, these are small and the variations much smoother than prior to UV illumination. Somewhat later (panel d) we can recognize the two +1 topological defects moving upwards towards the thinnest part of the shell. The steady-state texture is shown in Fig. 6e, where the two +1 defects have found their final positions. The fully planar alignment with a smoothly varying director field in the vicinity of the defects is confirmed by the variations in fluorescence intensity, reflecting the spatial variations in angle between director and laser polarization. At this point also a regular polarizing microscopy texture was recorded (Fig. 6f), showing a smooth nematic texture with the two +1 defects close to the thinnest point of the shell (the top), and with very little signs of phase separation. The dramatic difference from Fig. 6a shows that photoisomerization of the azo moieties of AzD not only has the ability to change the alignment but also to drastically improve the dissolution of poorly dissolved dendrimers. It is worth to mention that the 3D image of the upper half of the shell is not perfectly hemispherical, in contrast to the bottom half of the shell. This distortion of the shell shape in confocal microscopy results from the refraction of 

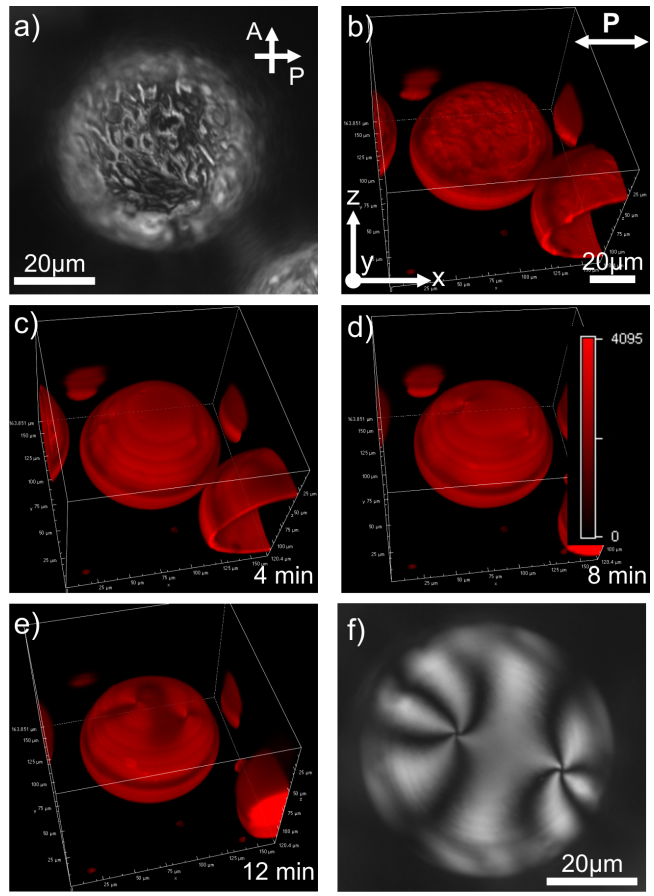

Figure 6: Nematic shell with 0.6 wt.- $\%$ AzD and 0.01 wt.- $\%$ Nile red before UV illumination imaged by POM (a) and FCPM (b). (c-e) FCPM images of the shell during UV illumination as a function of time. (f) POM image corresponding to (e).

the laser beam at the water-liquid crystal interface, due to its curvature and the high effective refractive index of the liquid crystal compared to water.

Apart from inducing motion of the shell during FCPM recording, the long-term UV illumination also has the unfortunate effect that it raises the risk of shell rupture. The shell depicted in Fig. 6 broke and collapsed into a droplet before the UV irradiation was turned off, hence we could not image its texture after the UV exposure. However, every investigated sample has many shells and they all respond qualitatively in the same way, hence we can study different shells before, during and after UV irradiation using FCPM. The results are summarized in movies SI4-5 and in Fig. 7, showing a 3D overview (first column) as well as horizontal slices at the top (column 2), equator (column 3) and bottom (column 4) of three different shells, each with 1 wt.- $\%$ AzD and 0.01 wt.- $\%$ Nile red. One shell was imaged before UV exposure (top row), one during exposure (mid row) and one was imaged after UV exposure (bottom row). 

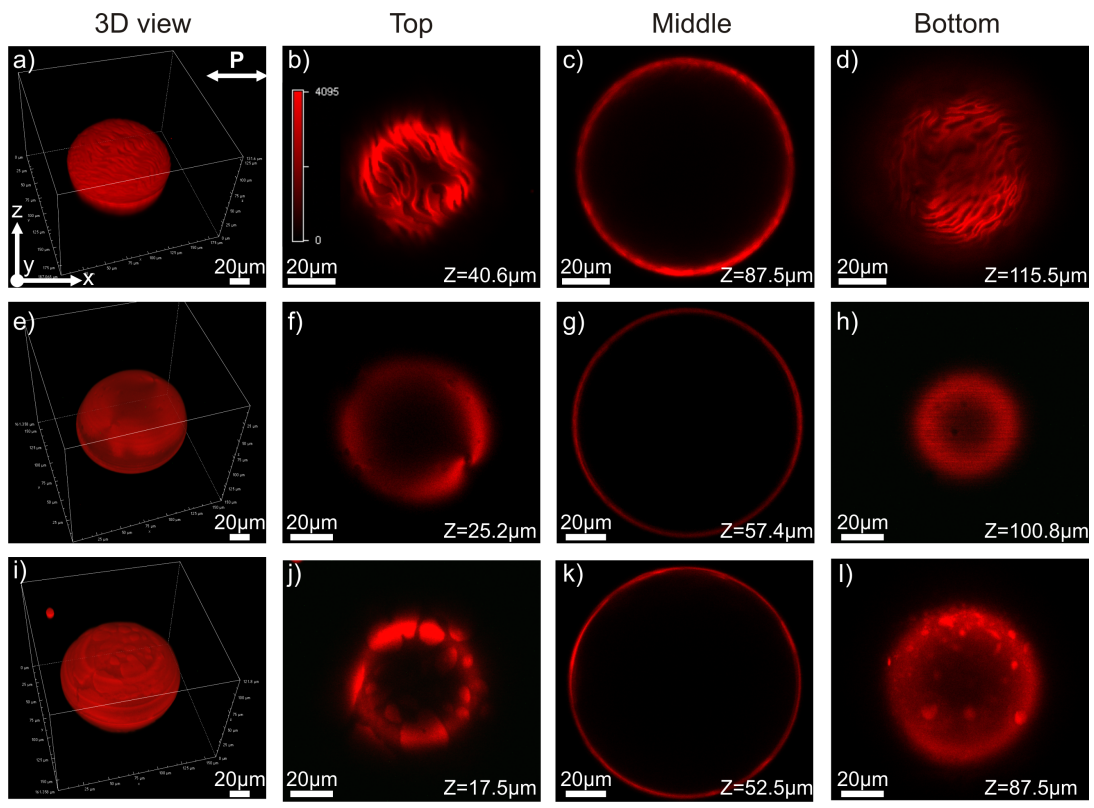

Figure 7: FCPM images of nematic shells with 1 wt.- $\%$ AzD and 0.01 wt.- $\%$ Nile red before (top row), during (mid row) and after UV illumination (bottom row). The first column shows the complete 3D images, the subsequent columns show slices at the shell bottom, equator and top, respectively.

Again the pristine shell shows phase separation on small scale, the texture characterized by wavy stripes. During UV exposure the shell looks largely uniform, topological defects being recognized near the top of the shell. The phase separation still returns after the UV exposure is turned off (Fig. 7i-l) but rather than stripes we see islands of segregated dendrimer. Moreover, these islands are very bright in the FCPM images, suggesting that the Nile red dye prefers to aggregate with the azodye motieties of AzD when the cistrans back isomerization takes place upon termination of the UV exposure.

\section{Conclusions and outlook}

We conclude that AzD is a highly useful surface-active agent for allowing rapid photoswitching of nematic liquid crystal shells, from fully homeotropic to fully planar. Since the effect is temperature-independent, it should be possible to apply it also on shells made from materials with a richer LC phase sequence, allowing alignment switching also in, e.g., smectic phases. 
If AzD-doped shells are made from a cholesteric with strongly temperaturedependent pitch, the effects of rapid alignment switching could be studied for long- as well as short-pitch cholesterics subjected to curved interfaces.

A non-planar ground state and an ideal switching behavior in the shells requires more than 0.3 wt.- $\%$ AzD and this must be very well dissolved. During UV exposure planar alignment is always promoted and the solubility of the AzD is enhanced in this state, even if it was initially phase separating. The choice of assistive solvent is surprisingly important for controlling whether or not phase separation occurs in the shell, considering that the solvent is evaporated prior to shell fabrication. Chloroform works very well, whereas dichloromethane yields phase separation in the ground state of shells made soon after mixture preparation.

\section{Acknowledgment}

We thank Anatoliy Gluschenko and Yuriy Garbowskiy for the opportunity to contribute to this special issue in memory of our dear friend Yuriy Reznikov. Financial support from the European Research Council under the European Union's Seventh Framework Programme (FP/2007-2013) / ERC Grant Agreement n.648763 (consolidator project INTERACT) is gratefully acknowledged. J.N. acknowledges support from the Fonds Nationale de la Recherche (FNR, Ph.D. grant ULISCO, code 6992111).

\section{Supplementary material}

Videos of photoswitching of 5CB shell with well dissolved AzD and with UV-induced removal of phase separation; two videos of FCPM-imaged shells (before and after UV exposure).

[1] M. Urbanski, C. G. Reyes, J. Noh, A. G. Sharma, V. S. R. Jampani, J. P. Lagerwall, Liquid crystals in micron-scale droplets, shells and fibers, J. Phys.: Condens. Matter 29 (13) (2017) 133003.

[2] A. Utada, A. Fernandez-Nieves, H. Stone, D. Weitz, Dripping to jetting transitions in coflowing liquid streams., Phys. Rev. Lett. 99 (9) (2007) 094502.

[3] T. Lopez-Leon, A. Fernandez-Nieves, Drops and shells of liquid crystal, Colloid Polym. Sci. 289 (4) (2011) 345-359. 
[4] A. Fernandez-Nieves, V. Vitelli, A. Utada, D. R. Link, M. Marquez, D. R. Nelson, D. A. Weitz, Novel defect structures in nematic liquid crystal shells, Phys. Rev. Lett. 99 (15) (2007) 157801.

[5] H. Liang, J. Noh, R. Zentel, P. Rudquist, J. Lagerwall, Tuning the defect configurations in nematic and smectic liquid crystalline shells., Philos. Transact. A Math. Phys. Eng. Sci. 371 (1988) (2013) 20120258.

[6] Y. Geng, J. Noh, I. Drevensek-Olenik, R. Rupp, G. Lenzini, J. P. F. Lagerwall, High-fidelity spherical cholesteric liquid crystal bragg reflectors generating unclonable patterns for secure authentication, Sci. Rep. 6 (2016) 26840.

[7] D. K. Kim, M. Hwang, J. P. F. Lagerwall, Liquid crystalfunctionalization of electrospun polymer fibers, J. Polym. Sci. B Polym. Phys. 51 (11) (2013) 855-867.

[8] E. Enz, J. Lagerwall, Electrospun microfibres with temperature sensitive iridescence from encapsulated cholesteric liquid crystal, J. Mater. Chem. 20 (33) (2010) 6866-6872.

[9] J. Wang, A. Jákli, J. West, Morphology tuning of electrospun liquid crystal/polymer fibers., ChemPhysChem 17 (19) (2016) 3080-3085.

[10] P. Cladis, A. White, W. Brinkman, The cholesteric defect structure near the smectic a transition, J. Phys. (Paris) 40 (3) (1979) 325-335.

[11] H.-L. Liang, S. Schymura, P. Rudquist, J. Lagerwall, Nematic-smectic transition under confinement in liquid crystalline colloidal shells, Phys. Rev. Lett. 106 (24) (2011) 247801.

[12] T. Lopez-Leon, A. Fernandez-Nieves, M. Nobili, C. Blanc, Nematicsmectic transition in spherical shells, Phys. Rev. Lett. 106 (24) (2011) 247802.

[13] D. Sec, T. Lopez-Leon, M. Nobili, C. Blanc, A. Fernandez-Nieves, M. Ravnik, S. Zumer, Defect trajectories in nematic shells: Role of elastic anisotropy and thickness heterogeneity, Phys. Rev. E 86 (2012) 020705(R). 
[14] J. Noh, K. Reguengo De Sousa, J. P. F. Lagerwall, Influence of interface stabilisers and surrounding aqueous phases on nematic liquid crystal shells, Soft Matter 12 (2) (2016) 367 - 372.

[15] M. Anyfantakis, D. Baigl, Dynamic photocontrol of the coffee-ring effect with optically tunable particle stickiness., Angew. Chem. Int. Ed. 53 (51) (2014) 14077-14081.

[16] H. Nádasi, R. Stannarius, A. Eremin, A. Ito, K. Ishikawa, O. Haba, K. Yonetake, H. Takezoe, F. Araoka, Photomanipulation of the anchoring strength using a spontaneously adsorbed layer of azo dendrimers., Phys. Chem. Chem. Phys. 19 (11) (2017) 7597-7606.

[17] W. Li, T. Dohi, M. Hara, S. Nagano, O. Haba, K. Yonetake, T. Seki, Phototriggered mass migration consorted with surface dewetting in thin films of a liquid crystalline azobenzene-containing dendrimer, Macromolecules 45 (16) (2012) 6618-6627.

[18] G. Lee, F. Araoka, K. Ishikawa, Y. Momoi, O. Haba, K. Yonetake, H. Takezoe, Photoinduced ordering transition in microdroplets of liquid crystals with azo-dendrimer, Part. Part. Syst. Char 30 (10) (2013) 847852.

[19] P. Hirankittiwong, N. Chattham, J. Limtrakul, O. Haba, K. Yonetake, A. Eremin, R. Stannarius, H. Takezoe, Optical manipulation of the nematic director field around microspheres covered with an azo-dendrimer monolayer., Opt. Express 22 (17) (2014) 20087-20093.

[20] A. Eremin, P. Hirankittiwong, N. Chattham, H. Nádasi, R. Stannarius, J. Limtrakul, O. Haba, K. Yonetake, H. Takezoe, Optically driven translational and rotational motions of microrod particles in a nematic liquid crystal., Proc. Natl. Acad. Sci. U. S. A. 112 (6) (2015) 1716-1720.

[21] I. I. Smalyukh, S. Shiyanovskii, O. Lavrentovich, Three-dimensional imaging of orientational order by fluorescence confocal polarizing microscopy, Chem. Phys. Lett. 336 (1) (2001) 88-96.

[22] K. Peddireddy, V. S. R. Jampani, S. Thutupalli, S. Herminghaus, C. Bahr, I. Muševič, Lasing and waveguiding in smectic a liquid crystal optical fibers, Opt. express 21 (25) (2013) 30233-30242. 
[23] T. Lopez-Leon, A. Fernandez-Nieves, Topological transformations in bipolar shells of nematic liquid crystals, Phys. Rev. E 79 (2) (2009) 021707 . 